\title{
Espace et communication réflexion sur le sentiment d'appartenance
}

Paul Stryckman

\section{(2) OpenEdition}

12 Journals

Édition électronique

URL : http://journals.openedition.org/communicationorganisation/1546

DOI : 10.4000/communicationorganisation. 1546

ISSN : $1775-3546$

Éditeur

Presses universitaires de Bordeaux

Édition imprimée

Date de publication : 1 mai 1992

ISSN : 1168-5549

Référence électronique

Paul Stryckman, «Espace et communication réflexion sur le sentiment d'appartenance »,

Communication et organisation [En ligne], 1 | 1992, mis en ligne le 26 mars 2012, consulté le 19 avril

2019. URL : http://journals.openedition.org/communicationorganisation/1546 ; DOI : 10.4000/

communicationorganisation. 1546

Ce document a été généré automatiquement le 19 avril 2019.

(c) Presses universitaires de Bordeaux 


\title{
Espace et communication réflexion sur le sentiment d'appartenance
}

\author{
Paul Stryckman
}

« On sait maintenant que l'espace social est un système de communication quand il est investi par le sens et les sens qu'une communauté réelle prend de l'existence et de ses divers aspects. » R. Ledrut.

\section{Espace et communication}

\section{Espace}

1 Espace est un concept polysémique. En effet, que faut-il entendre par espaces économiques (Boudeville, 1961), espaces médiatiques (Garnier, 1987), espaces culturels (Delcourt, 1991), espaces privés/publics...?

2 Comme point de départ, distinguons trois sens au concept d'espace. L'espace est le lieu ou étendue en tant que réalité «locale », c'est le «lieu» d'une mesure. Dans ce sens, l'étendue du mètre est invariante. On peut aussi s'entendre sur l'espace d'une «ville moyenne " tant dans son aire géographique que dans son importance démographique. Mais l'espace devient un territoire dans la mesure où il est le lieu d'une transaction sociale, où il est marqué de rapports avec autrui, investis de juridictions. Ainsi, le mètre reçoit une signification nouvelle dans l'établissement du «territoire personnel » comme extension du corps. L'espace territoire est nécessairement orienté : il est généralement identifié par un centre et délimité par un périmètre plus au moins précis. L'espace est un territoire investi de représentations symboliques, de valeurs, de normes, de codes d'échanges. Dans ce sens, parler de la ville moyenne, du quartier, de la rue, de la maison, de l'appartement, c'est aussi évoquer un mode d'être, un ensemble de significations spatialisées En d'autres mots, ils deviennent plus que des lieux et des territoires ${ }^{1}$. On peut dire que les espaces urbains et ruraux peuvent dénoter des mêmes réalités mais avec des 
connotations sémantiques différentes ${ }^{2}$. Ce sont des espaces symboliques dans un double sens : d'une part, ce sont des espaces concrets (lieux et territoires) investis de symboles qui permettent à chacun de se situer dans une vision concrétisée du monde, d'autre part, ce sont aussi des espaces-concepts avec lesquels on peut penser, se «figurer " des échanges, des modes d'être et de devoir être. C'est pourquoi, il est possible de penser résidence, quartier, ville, centre-ville, village en voyant dans ces " espaces » des types spécifiques de relations avec autrui et la nature, des modes de communication.

Parler de l'espace urbain sans tenir compte de ces distinctions, c'est rester prisonnier de la polysémie et entretenir la confusion. Vouloir penser autrement la ville, ce n'est pas ignorer les sérieuses contraintes des lieux physiques ni les forces sociales qui caractérisent ses territoires, c'est aussi communiquer de nouvelles pratiques sociales ou de nouveaux modes culturels. C'est bien à ce constat qu'arrive Raymond Ledrut quand il dit dans L'espace en question:

«La ville est un lieu de signification. Elle est donc un moyen de communication. (...) La communication par l'espace social et ses structures ne peut s'établir qu'à partir d'une symbolique culturelle commune. Et ce qui est important c'est que cette symbolique n'est pas fondée sur un code : la signification suppose le sens et non le sens la signification. (...) La communication par l'espace social ne suppose donc pas l'acquisition d'une même langue ou d'un même code, qui établiraient des relations conventionnelles et fixes entre unités discrètes du signifiant et du signifié; elle exige la possession d'une même "vision du monde » (Ledrut, 1976 : 215)

4 Mais alors la question à se poser est de savoir s'il y a une vision commune du monde urbain ? Y a-t-il un langage commun sur les structures et les pratiques urbaines? Non pas un langage inscrit dans le bâti urbain à "communiquer » à tous les occupants, mais un même langage sur, un même investissement affectif dans, une même appropriation symbolique des espaces urbains par la population résidente. L'erreur du professionnel de la communication publique, de l'administrateur municipal, de l'urbaniste visionnaire ne serait-elle pas de penser et de travailler avec un seul langage, une seule vision urbaine du monde et de vouloir l'imposer normativement en sous-estimant les fonctions structurantes des langages urbains déjà pratiqués par les occupants.

\section{Communication}

5 Il fut un temps où le terme communication avait comme premier sens celui de partageavec, il appartient alors à la même racine sémantique que le terme " communion ", c'està-dire de mise en commun (mettre en commun). Au 16e siècle, il prend le sens restrictif de faire part(age) d'une nouvelle, de transmettre et il se produit un glissement de sens du « partage » à la «transmission » et aux moyens de transmission qui sont des moyens de communication ${ }^{3}$. Les communications vont appartenir aux ingénieurs friands d'un monde linéaire, construisant trains, télégraphes, ponts et chaussées. Cette acception connaîtra son apogée avec le binôme paradigmatique émetteur/récepteur et ses multiples variantes. On peut facilement comprendre le succès d'un tel binôme partout où il faut assurer la diffusion d'idées, de priorités, de politiques. La communication municipale n'ignore pas la réalité politique de ce binôme !

Mais le simplisme efficace du modèle linéaire s'est avéré insatisfaisant pour rendre compte de tous les phénomènes de communication. Des chercheurs sont venus, par des cheminements différents, au sens premier de la communication comme ensemble, comme système de codes ou d'informations activées inconsciemment dans la mise en commun 
d'êtres humains. Dans cette perspective, un système de communication équivaut pratiquement à un système culturel dont les règles inconscientes permettent des échanges complexes. On pourrait pousser jusqu'à dire que l'analyse de la communication devient alors une recherche sur le mode d'être par le dévoilement des codes fondant et permettant les échanges entre des «je/nous», des «tu/vous", des «ils/eux». Comme l'espace peut justement être lui-même le «lieu » de codes : il est inscription de codes, il est sens. Mais comme support d'interactions symboliques, il devient langage et communication.

7 Insistons sur le statut inconscient de ces codes culturels. C'est comme si les relations avec les autres dans un espace donné, l'aménagement de l'espace forment des langages et que ces derniers marquent et actualisent des identités culturelles ${ }^{4}$. Dans ce sens, il faudrait pratiquer une analyse sémantique à la fois des espaces aménagés comme langages vécus et des langages parlés qui nomment et connotent le village, la ville, l'agglomération, la communauté urbaine.

\section{Le sentiment d'appartenance}

8 Nous cernons cette notion par les apports théoriques de la sociologie des groupes et de l'anthropologie culturelle.

\section{La sociologie des groupes}

9 Cette théorie permet de distinguer deux notions importantes dans le traitement de l'espace par l'individu. Il s'agit du sentiment d'appartenance vécu par les membres d'un groupe et les mécanismes de référence qu'un individu peut manifester à l'égard d'un groupe.

$\mathrm{Du}$ point de vue individuel, le sentiment d'appartenance entretient des attitudes et programme des comportements par lesquels le citoyen s'identifie, établit des préférences, oriente ses motivations, participe à des activités. Tout cela lui donne un sentiment de rattachement, voire d'engagement. Du point de vue de l'action sociale, l'appartenance exige à la fois un minimum d'interactions entre l'individu et le ou les groupes et l'actualisation de normes et de valeurs propres aux groupes. La proximité spatiale, ce qui pourrait être traduit par des relations de voisinage, par exemple, peut imposer des codes formels et informels d'appartenance au quartier dans l'aménagement résidentiel [règlements d'urbanisme] et l'entretien des propriétés 5 . Il saute aux yeux que le développement social, ce qui dans notre cas se traduit par le développement urbain, peut entraîner la multiplication et la différenciation des groupements intermédiaires produisant des mutations dans les sentiments d'appartenance au point de générer des investissements affectifs et symboliques différenciés, des distances sociales, des appartenances oppositionnelles ${ }^{6}$. Ce développement peut aussi produire un vide social et des situations anomiques. N'est ce pas pour cela qu'il est communément dit que la vie municipale est la base de la démocratie ${ }^{7}$ ?

11 La ville n'est pas réductible à une réalité sociologique analysable par la théorie des groupes aussi complexe que celle-ci puisse devenir pour la simple raison que sa première vérité est un espace territorial, lequel peut être investi d'actions politiques, économiques 
et sociales, mais c'est avant tout un espace comme « lieu premier » d'interactions parce qu'espace habité sur lequel on vit.

Nous venons de conjuguer appartenance et identification. Cette dernière notion exige une précision importante parce qu'un groupe de référence peut remplir deux fonction fort distinctes. La première fonction est normative : l'individu cherche à se faire accepter pour appartenir au groupe. À toute fin pratique, le groupe entretient alors une référence normative d'identification inculquant des standards de conduites et d'attitudes. Mais le groupe peut aussi être un simple point de référence, c'est un groupe étalon que l'individu utilise pour se former des jugements et des évaluations sur soi ou sur autrui. Le groupe remplit ainsi une fonction comparative utilitaire sans retombée d'appartenance, il est réduit à une référence identitaire n'infléchissant aucun comportement ni attitude. En d'autres termes, la référence devient un élément d'identification sous un double mode : un mode motivationnel qui explique la source de normes régulatrices et entretient cellesci, c'est l'identification forte, et le mode perceptuel où le groupe n'est qu'un référent parmi bien d'autres, c'est l'identification nominale.

La référence oppositionnelle peut produire des effets d'appartenance construisant des « nous » rhétoriques par lesquels le nous-Bordeaux s'oppose à Paris, le nous-Québec ville se monte contre Montréal, le nous-Québec capitale, contre Ottawa...

On peut dégager un enseignement utile pour cette approche de l'espace sous l'angle de la théorie des groupes. Il y a des territoires à identification nominale, instrumentale, qui peuvent être de l'ordre du classement conventionnel : la résidence est réductible au code postal ou au code numérique d'une "ligne» téléphonique pour le moment encore spatialisée. Il y a des territoires appropriés pour réaliser des «nous » et auxquels, par paradoxe rétroactif, nous appartenons. Le domicile, la rue, le quartier peuvent remplir d'importantes fonctions d'identification forte parce qu'ils sont des "milieux de vie" mobilisant et activant des codes d'appartenance.

\section{L'anthropologie culturelle}

15 L'anthropologie culturelle présente l'identité comme un processus d'intériorisation d'une image de soi comme personne et comme acteur social par rapport à autrui et à différentes activités et situations de la vie quotidienne. On peut dire que l'identité personnelle repose sur l'assomption de modèles de pensée et de comportements caractérisant des modes d'être en société, c'est l'intériorisation de modèles collectifs intégrés. Mais il y a identité dans la mesure où il peut y avoir repérage de la différence, lequel peut même s'inscrire dans le traitement des différences. Le traitement de la différence comme facteur identitaire n'est nullement étranger à l'aménagement de l'espace. Les rapports à l'espace sont intimement liés à une vision du monde et à des paramètres de «spatialisation du moi ». L'intériorisation de valeurs et de normes fournit à chacun des grilles de lecture pour le décodage tant du langage, du temps et de l'espace (Remy, alii, $1978: 336 s s$.$) .$

Mais il ne s'agit pas d'une simple «représentation » de soi, cette image entretient aussi un investissement affectif procurant une sécurité et une stabilité psychologique qui se permet de situer par rapport à autrui dans un espace et un temps donnés. Dans ce sens, nous allons parler d'une appropriation de l'espace. Faisons remarquer qu'il n'est pas question ici de groupes, mais de schèmes mentaux articulant des langages de la vie 
quotidienne. Cette approche s'applique donc parfaitement à l'analyse de l'identité et de l'espace.

17 Nous savons que le comportement de l'individu traite l'espace selon des règles inconscientes: il communique aussi par un langage de l'espace. Il ne s'agit pas de développer ici la proxémique de Hall et sa typologie des territoires (Hall, 1981). Mais l'individu se positionne différemment dans un espace social en fonction de paramètres structurant son identité sociale. Ainsi, peut-on distinguer un rapport concret d'un rapport abstrait avec l'espace. Dans un rapport concret à l'espace, les membres d'un groupe retrouvent leur identité et leur sécurité sur un territoire organisé par des objets concrets et des localisations inscrites dans la mémoire collective du groupe. Dans un rapport abstrait à l'espace, les membres d'un groupe peuvent retrouver leur identité et leur sécurité dans un territoire étranger dans lequel ils peuvent retrouver des homologies spatiales et des localisations de substitution grâce à un processus de "dématérialisation " de l'espace: ils opèrent plus avec des structures significatives qu'avec des objets, plus avec des signifiés que des signifiants ${ }^{8}$.

Le rapport concret réduit les possibilités de mobilité spatiale et tend à maintenir un lien d'appartenance à un territoire immédiat et son bâti. Il va fortement valoriser des relations à l'intérieur du territoire familier et entretenir des rapports d'opposition avec l'extérieur perçu comme un danger, un risque de perte d'identité.

\section{Espace et appartenance}

\section{Types d'appropriation de l'espace}

L'inscription de l'appartenance dans l'espace peut être approchée par les rapports entre l'appropriation de l'espace et la formation de l'identité à la fois individuelle et collective. Nous simplifions cette approche en relevant quatre rapports typiques qui déterminent quatre espaces.

20 L'espace éphémère des populations nomades, c'est un espace-sol jalonné des marques de l'itinérance. Attention, nos grandes villes abritent une population nomade de mutants et de sans-abris.

21 L'espace-terroir des populations agraires qui inscrivent spatialement leurs rapports socio-économiques et politiques, ce sont des terres d'appartenance des sociétés paysannes.

22 L'espace-ville différentiellement approprié selon le développement de la division sociale du travail. C'est originellement l'espace-rue en tant que lieu de la mixité en territoires unifonctionnels de production, distribution, consommation, loisirs. C'est un espace quadrillé par des quartiers, des zones entre lesquelles les occupants, redevenus nomades urbains, circulent entre l'habitation, le travail, le marché, le jeu sur des territoires où tout devient affaire de rencontre Duvignaud, 1977 : 31-38).

L'espace-réseau de la transnationalisation marchande qui n'est plus soumise aux contraintes du sol, ou du terroir rural ni aux juridictions des territoires urbains et nationaux. Cet espace n'est plus cumulatif ou extensif comme celui de la métropole ou de la mégapole, il devient système ouvert, support dématérialisé de flux d'informations et d'échanges. 
24 L'appropriation de l'espace transforme des espaces concrets dans des oppositions symboliques structurant l'identité des «occupants». Mentionnons au passage les rapports

- du dedans [du village, du quartier]/ du dehors [de la ville],

- du centre [de l'ordre]/

- de la périphérie, banlieue [désordre, violence],

- du passé [à préserver, rénover]/ de la modernité [à « promouvoir »],

- de l'Est [de la rue médiane]/ de l'Ouest [de la rue médiane],

- du Nord [de la voie ferrée]/ du Sud [de la voie ferrée],

- du Nord [hémisphère riche] du Sud [hémisphère pauvre],

- du haut [haute ville]/ du bas [basse ville],

- de la rive droite [échec]/ de la rive gauche [réussite]

Ces rapports peuvent être vus comme des dimensions structurant des lieux. Les situations concrètes sont généralement investies de plusieurs dimensions. Ceci peut avoir comme effet symbolique de produire des espaces urbains complexes. Il faut ajouter que ces codes spatiaux visualisent et démarquent toujours des intérêts individuels et collectifs. Mais leur lecture et leur investissement ne sont pas nécessairement les mêmes pour tous les habitants. Dans ce sens, il serait intéressant d'étudier comment les occupants d'un espace métropolitain sémantisent ce dernier. Il ne serait pas étonnant qu'une enquête révèle ainsi l'existence de centres urbains distincts, l'image d'un espace polycentré affecté de sentiments d'appartenance et de référence pluriels.

Ainsi, en milieu rural proche d'une agglomération urbaine, un même lieu peut être un milieu de vie traditionnelle, un lieu résidentiel (les villes ou villages dortoirs), un lieu de détente en fin de semaine ou de vacances. Ces appropriations de l'espace rural ne produisent ni ne reproduisent pas un même sentiment de solidarité et d'appartenance : pour les uns, il est enracinement [infra], pour d'autres, point de chute provisoire, lieu de passage pour d'autres. Dès lors, comment générer un sentiment d'appartenance?

\section{L'appartenance en milieu urbain}

La situation se complique en milieu urbain. L'occupation d'un même espace, même un bloc d'appartements' ${ }^{9}$, ne signifie pas la formation d'une même solidarité ni l'entretien d'un même sentiment d'appartenance. Les uns veulent un "bon" quartier en revendiquant un espace tranquille avec l'école proche, le terrain de jeux, le centre de loisirs... Ils veulent recréer un milieu de vie ; les autres se contentent d'un logement, d'un appartement à moindres frais, les services étant disponibles ailleurs. Les uns s'approprient un espace en le voulant multifonctionnel par l'aménagement d'un village ou d'une "petite ville» dans la ville. Les autres s'approprient toute la ville: ils se perçoivent clients de ses services, des ses territoires. Les uns s'identifient à leur quartier. Les autres vivent leur ville avec ses lieux d'échanges et ses ambiances diverses où ils « se retrouvent", ils développent leur identité dans une pluralité de lieux ${ }^{10}$. Deux consommations de l'espace urbain, deux représentations bien différentes des mêmes espaces et des équipements urbains, notamment des centres urbains.

Par analogie, l'espace urbain peut être vu comme le lieu du clivage de multiples marchés (l'habitat, l'emploi, le commerce, le loisir, l'instruction.) façonnant des lieux fonctionnels et des lieux de vie. Dans une perspective fonctionnaliste, la ville perd sa mixité fonctionnelle unicentrée garantissant une référence identaire pour détenir le monopole 
de produire et de reproduire par la diversité de ses espaces et de ses équipements un éclatement polycentré de sociabilités, de réseaux et d'ambiances que des clientèles résidentes ou passagères s'approprient différentiellement selon des codes de comportements sociaux et de normes culturelles fort différents.

Le quartier urbain, le village rural peuvent être des espaces du dedans formant des territoires d'appartenance auxquels l'occupant s'identifie, se rattache, pour lesquels il est prêt à engager des actions. La grande ville, l'agglomération urbaine sont des lieux de référence, sur lesquels les médias émettent des codes, annoncent des politiques, dictent des langages, des conduites, font infléchir des attitudes.

En deçà d'un seuil de croissance, la ville est une cosmologie habitée et définie par des tracés territoriaux : la rue et l'agora, la grande place, lieux publics, topiques de la liberté et de l'égalité dans la diversité, définissent une image de l'humanité urbaine capable de confronter la différence dans la coexistence ${ }^{11}$. La ville gère ainsi la mixité et l'aléatoire (Schoonbrodt, 1987).

Mais dès le dépassement d'un seuil d'accroissement urbain, il n'y a plus un domaine ni un territoire, mais des territoires unifonctionnels où l'on travaille ici, se divertit là, vient dormir là-bas... L'habitant y devient alors le nomade entre plusieurs territoires. La ville se transforme en un espace polycentré. L'évolution démographique et le développement technologique produisent une décentration du noyau urbain à tel point que la rue est principalement perçue dans sa fonction circulatoire productrice de "chronophagie » (Robert, 1980). Finalement l'ancienne ville, toujours nommée centre, est ceinturée d'un circuit, d'un périphérique, d'un « ring », conquis par l'automobile évitant le centre-ville devenu inaccessible au nomade motorisé.

\section{Appartenance et histoire}

Il faut compléter notre réflexion en relevant une dimension particulière de l'espace local, c'est celle de domaine parce que l'espace doit être géré à la fois dans le quotidien et dans la tradition. Le local est aussi le territoire investi d'une mémoire collective qui le transforme en patrimoine. Il ne peut donc pas être géré comme propriété privée parce qu'il est aussi le lieu d'« un legs des générations passées aux générations futures ». Le local s'inscrit ainsi dans une perspective de filiation et de mémoire proprement patrimoniale (Gaudin, 1989 : 62-63). En d'autres mots, l'espace local est aussi le lieu d'un enracinement de l'histoire et d'une mémoire collective. Le sentiment d'enracinement requiert l'existence d'un groupe social relativement stable et un ensemble bâti offrant des points de référence durables comme les places de rassemblement, les édifices, les monuments, vus comme des inscriptions de l'histoire dans le local. Dans ce sens, l'expérience individuelle et collective de la durée viennent qualifier l'enracinement: l'espace devient aussi le lieu du temps historique.

Les notions de filiation, de patrimoine et de domaine soulèvent la question du rapport entre le sentiment d'appartenance et de l'histoire. Il n'y a pas simplement une appropriation psycho-sociale de l'espace telle que le laisserait penser notre réflexion basée sur l'utilisation de codes culturels, il y a aussi l'appropriation institutionnelle découlant de la gestion du domaine local par les autorités locales qui sont tout à la fois gestionnaires d'un héritage, de problèmes du présent quotidien et "promoteurs » d'un avenir. On peut voir poindre ici, dans notre réflexion, toute la réalité de la dynamique municipale et sa gestion des sentiments d'appartenance et de référence ${ }^{12}$. 

les rapports du local à l'histoire. Il est intéressant de noter comment, dans certaines municipalités de banlieue, les résidents qualifient encore aujourd'hui une aire centrale de « village » comme si la nappe urbaine n'avait pas encore complètement couvert l'histoire du premier bâti rural, comme si l'ancienne aire agricole de la municipalité « résidentialisée » par des promoteurs urbanisant a perdu toute histoire, toute vertu de filiation patrimoniale. L'espace urbain "pavillionnaire» troque la modernité fonctionnaliste contre toute appartenance à l'histoire.

Mais il n'y a pas que le développement domiciliaire qui génère des territoires sans histoire, la mobilité de la population urbaine combat des effets de durée. Il faut alors produire une histoire en soulignant les dates de fondation, la valeur patrimoniale d'un moulin, d'un pont, d'un manoir, d'une maison, en joignant le ludique ou l'onirique à l'histoire ${ }^{13}$. On doit se demander si le fait plutôt exceptionnel aujourd'hui de la longue durée résidentielle en milieu urbain n'a pas tendance à reporter pour l'essentiel le travail sur la mémoire sur les signes spatiaux. Manque de temps dans la mémoire collective, on se met à marquer l'espace (Gaudin, 1989: 67). C'est le développement d'un dispositif de marquage territorial et institutionnel qui va principalement se concrétiser par des politiques dans l'agencement d'équipements collectifs et de services qui «encadrent, domestiquent » l'animation socio-culturelle et les formes de socialité locale ${ }^{14}$.

\section{Appartenance et communication}

\section{Le quartier et la communauté perdue}

Durant notre parcours, nous avons souvent rencontré l'espace-quartier. En traitant du sentiment d'appartenance, il est impossible de ne pas s'y arrêter quelques instants. On pourrait le faire en nous demandant si le quartier urbain ne serait pas l'urbanisation du concept de communauté ? Dans la tradition sociologique, la communauté locale est caractérisée par trois composantes principales: des réseaux de relations interpersonnelles extérieures au foyer, un lieu de résidence commun, des sentiments et des activités solidaires (Wellman, Leighton, 1981 : 112).

La tradition sociologique manifeste une réelle obsession de la communauté comme expression de l'intégration sociale et du consensus social que l'urbanisation industrielle et les processus bureaucratiques modernes ont gravement déstructuré pour produire l'homme urbain individualiste, compétitif, étranger de la vie de groupe. C'est le paradigme de l'anomie urbaine qui peut être corrigée par la multiplication des réseaux de communication produisant une «solidarité technologique » de la télécommunication et par le social virtuel distribué par les mass-médias. Dans cette perspective, le quartier urbain a reçu le quasi-monopole de réalisation des sentiments de solidarité à cause des vertus des relations de voisinage (1964. La cité à travers l'histoire. Paris : Le Seuil. Muret, J.-P.JS 4895 D884.1978. 1989. Informer sur la vie municipale, Paris: Éditions du CFPJ. Noschis, 1984). Mais affirmer que le quartier est le cadre de liens intra-communautaires, c'est affirmer l'a priori du pouvoir organisateur de l'espace, c'est confesser un déterminisme spatial, c'est prétendre que la cohabitation résidentielle doit produire des solidarités. Un quartier sans indice suffisant de vie communautaire engage des réflexes d'action communautaire comme si le quartier avait le monopole de la communauté, comme si la vie communautaire n'était pas viable ailleurs et autrement dans l'espace 
urbain. La résidence et la solidarité ont favorisé l'identification de la communauté au quartier comme si la communauté de réseaux complexes et les solidarités des relations de travail ne méritaient aucune attention.

L'obsession de la communauté perdue a hautement valorisé le quartier comme unique espace urbain à densité humaine à protéger et à défendre. Il ne faut toutefois pas oublier que le quartier en milieu urbain est aussi un quart de vie (Beringuier, 1980)... Il est grand temps d'entreprendre un examen serré du langage normatif qui lie quartier et communauté, langage qui donne souvent appui à des entreprises municipales sans succès.

\section{L'espace et son traitement médiatique.}

Dans Les métamorphoses du futur, Thierry Gaudin (1988) avance que :

«On passe d'une société où l'information était bien rare à un système de surinformation. La demande essentielle n'est plus la demande d'information, mais de sens, de grilles de lecture.» (p.41). Une réflexion globalisante montre que le phénomène déterminant de cette métamorphose est l'émergence d'un nouveau territoire social, après celui de la terre, puis du capital, le territoire du mental. C'est en lui que se situent désormais les nouveaux modes de production et surtout les nouveaux enjeux de pouvoir. » (p. 54) (Gaudin, 1988:54)

«Le mental ne se réduit pas à la seule intelligence. Il est le lieu où l'être individuel se construit et se développe par ses échanges avec les autres et avec le monde, le terrain nourricier du rêve et du spirituel. » (1988:56) (Gaudin, $1988: 56)$

Comment faut-il réagir devant une telle prospective quand nous savons aussi combien l'urbanisation des populations sera croissante dans les décennies à venir?

Comment donner sens et quelle grille de lecture faudra-t-il donner aux espaces urbanisés que nous savons maintenant lieux de langages bâtis et de langages parlés ? L'idée de grille de lecture nous invite à l'élaboration de tout un programme qui donnera à l'espace des significations.

Dans un tel contexte, les pratiques médiatiques peuvent remplir plusieurs fonctions en tablant sur cette diversité de significations. On pourrait commencer par établir un programme d'évaluation des informations locales traitées médiatiquement (Regazzola HT 101 E77 50-51 1987 ; Lauraire, R., Briole A., 1987).

Ainsi les contenus des textes médiatiques peuvent être :

- signalétiques : informations ponctuelles et souvent codées concernant des faits éphémères, périodiques pouvant meubler la vie quotidienne (ex. horaires, tarifs, calendriers, index, cotations...)

- événementiels : comptes rendus d'événements survenus dans l'espace local où le territoire est simple support matériel servant à la sélection des « récits » (stories) à rapporter. Le cas le plus typique est certainement le fait divers qui devient « nouvelle » par sa seule vertu d'être survenu sur le territoire.

- problématiques : positions d'un problème ou d'une problématique portant sur le territoire ou sur un segment de celui-ci. Le texte prend ici le territoire comme objet d'information critique et d'analyse et non plus comme simple support de l'information. Ici le territoire devient l'enjeu d'une réflexion, d'un débat, d'un conflit. Il n'est pas réduit au lieu du spectacle local, il s'affirme comme lieu d'une convivialité. pour traiter journalistiquement les enjeux territoriaux et socio-culturels d'un espace. Ici 
le territoire devient ainsi l'enjeu d'une réflexion, d'un débat, d'un conflit pour lequel le pouvoir local n'est qu'un acteur parmi bien d'autres. L'espace cesse d'être un lieu de référence ou l'occasion d'un récit signifié par la proximité spatiale, il se pose comme image et signification à donner à un projet et un mode de vie.

\section{BIBLIOGRAPHIE}

Auriac, F., Brunnet R., « Espaces, jeux et enjeux », Fondation Diderot/Fayard, 1986.

Battle, A., Les travailleurs du futur, Seghers, Paris 1986.

Beaunez, R., Kohn, F., La démocratie locale. Un préalable : l'information dans la cité. Éditions Ouvrières, Paris, 1975.

Becker, T., Scarce, R., "Why teledemocracy grows in America », dans Dervin B., Voigt M.J., Progress in communication sciences, volume VIII, Norwood N.J., 1975

Beringuier, C, « Se reconnaître dans l'espace de la ville : à chacun son quartier ! (Identité collective et quartier)», Espaces et Sociétés, 34-35: 75-82, 1980.

Boudeville, J-R., Les espaces économiques, Presses Universitaires de France, QSJ Paris, 1961

Castells, M., La question urbaine,Maspero, Paris 1972.

Cauquelin, A., Essai de philosophie urbaine, PUF,Paris 1982.

Chamboredon, J.Cl., Lemaire, M. « Proximité spatiale et distance sociale. Les grands ensembles et leur peuplement », Revue Française de Sociologie, XI : 3-33, 1970.

Choay, F., (éd.), Le sens de la ville, Le Seuil, Paris 1976.

Delcourt, J., « Marchandisation de la culture et nouveaux espaces culturels », Contradictions, 64 : 45-66, 1991.

Colloque de Cerisy, Métamorphoses de la ville,, Economica, Paris 1987.

Colloque international de Lyon HT 107 C7142.1983

L'idée de la ville, Champ Vallon, Presses univesitaires de France, Paris 1983.

de Tocqueville A., De la démocratie en Amérique, 10/18, Paris 1970.

Esgagnes. P., « Les obligation faites aux municipalités en matière d'information », Municipalités, Janvier : 31-32, 1986.

De Roux, E., «La politique d'aménagements des jardins. Des paradis retrouvés, Le Monde, 23 mai, p. 17, 1991.

Dulong R.,, Les régions, l'État et la société locale, PUF, Paris 1978.

Ferrarotti, F. The end of conversation. The impact of mass media on modernsociety, New York 1988. Greenwood.Garnier, J.-P. «L'espace médiatique ou l'utopie localisée », Espaces et sociétés, 50 : 7-20, 1987.

Gaudin,P., Technopolis. Crises urbaines et innovations municipales, PUF, Paris 1989. 
Gaudin,Th., Les métamorphoses du futur, Économica, Paris 1988.

Guéry, L., Quotidien régional, mon journal, Centre de formation et de perfectionnement des journalistes, Paris 1987.

Habermas, J., Théorie de l'agir communicationnel, 1987.

The structural transformation of the public sphere. An inquiry into a category of bourgeois society, The MIT Press., Cambridge Mass. 1989.

Hall, E.T., Au-delà de la culture, Le Seuil, (orig. : Beyond culture, 1976, NY), Paris 1979.

"Proxémique », dans Bateson G., alii, La nouvelle communication, Textes recueillis et présentés par Y. Winkin, Le Seuil, 191-221. (orig. 1968), Paris 1981.

Lassus, B., « Les jardins des Songes », Temps Libre I,

« La mobilisation au niveau local et la politique du système mondial »,

Revue internationale des sciences sociales, août : 381- 402, 1988.

Hiernaux, J.P., J. Remy, « Rapport à l'espace, rapport au corps et intégration sociale », Recherches sociologiques, $\mathrm{VI} / 3: 321-332,1975$.

Ion, J., A.Micoud, « La commune entre l'État et le quartier. Quelques notes sur l'évolution des types de légitimation de la pratique politique municipale », Espaces et Sociétés, 34-35, 1979.

Josephe, P., Muret, P., L'information-communiquer avec les citoyens dans la commune, Syros, Paris 1983..

Kaes, R., Vivre dans les grands ensembles, Éditions Ouvrières, Paris 1963.

Kelley, H.H., « Deux fonctions des groupes de référence », dans Lévy, A. : 139-145, 1978.

Lauraire, R., Briole A., "L'espace médiatique et le territoire », Espaces et sociétés, 50 : 151-172, 1987.

Ledrut, R., L'espace en question, Éditions Anthropos, Paris, 1977.

Marie, J-L., «La symbolique du changement », dans Mabileau A., Sorbets C, Gouverner les villes moyennes, Centre d'étude et de recherche sur la vie locale, Série Vie Locale No 12, PEDONE : 109-149, 1989.

Meier, R.L., Croissance urbaine et théorie des communications, PUF, Paris 1972.

Mucchielli, R., Le mythe de la cité idéale, PUF, Paris 1960.

Mumford, L., La cité à travers l'histoire, Le Seuil, Paris 1964..

Muret, J.-P., Informer sur la vie municipale, Éditions du CFPJ, Paris 1989.

Noël, P., Le tambour de ville, ou comment l'administraion écoute, renseigne, informe, La documentation française, Paris 1982.

Noschis, K., Signification affective du quartier, Librairie des Méridiens, Paris 1984.

Poche, B., «La région comme espace de référence identitaire », Espaces et Sociétés, 42 : 3-12, 1983.

Quesnel, L., « La démocratie municipale au Québec », Politique, 9 : 61-97, 1986.

Rabaté, Fr., « La production « locale » de l'audiovisuel », Espaces et sociétés, $50: 24-66$.[Bib.], Paris 1985.

Rabasté, Fr., (dir), Médias et changements sociaux, La Documentation Française, Paris 1985.

Regazzola, Th., « Médias, contenus, territoire », Espaces et sociétés, 50 : 69-94, 1987. 
Ramonet, I., « Reconstruire », Le Monde Diplomatique, sep-tembre, No 450, 1991.

Rémy, J. « Espace et théorie sociologique. Problématique de recherche ", Recherches sociologiques, $\mathrm{VI} / 3: 279-293,1975$.

Remy, J., L. Voyé, Ville, ordre et violence, PUF, Paris $1981 .$.

Remy, J., L. Voye, E. Servais, Produire ou reproduire? Tome I : Une sociologie de la vie quotidienne, Vie Ouvrière, Bruxelles 1987.

Robert, J., Le temps qu'on nous vole : contre la société chronophage, Le Seuil.Schoonbrodt, R., Paris 1980.

Essai sur la destruction des villes et des campagnes, P. Mardaga Bruxelles 1987.

Vermeylen, P., "L'extension de la sphère marchande et l'aménagement du territoire », Contradictions, $64: 69-79,1991$.

Voyé, L., J. Remy, « Scénarios de vie sociale. Analyse des effets de milieux au centre ville », Recherches Sociologiques, VI/3 : 294, 1975.

Wellman, B., B. Leighton,

« Réseau, quartier et communauté. Préliminaire à l'étude de la question communautaire », Espaces et Sociétés, 38-39: 111-133, 1981.

« Sociologie du « local » et « relocalisation » du social », Sociologie du Travail, No 2, 1983.

«L'espace institutionnel et la dimension du local », dans Rabaté F., Medias et changements sociaux, 115-126, 1985.

Voix de la politique locale, Mots. Les langages du politique, $\mathrm{N}^{\circ}$. 25. Rey H., Dire le local en banlieue : 105-108, 1990.

\section{NOTES}

1. Encore faudrait-il se méfier des résidus sémantiques avec lesquels le langage ordinaire charge ces termes!

2. Faisons remarquer que le terme espace est lui-même valorisant par ses connotations positives, ce que le concept d'étendue n'implique pas.

3. C'est assez récemment que les instances gouvernementales ont distingué la gestion des transports et des communications.

4. Analysant la dernière révolution russe, I. Ramonet note que le régime soviétique « minimisait les autres attributs de l'identité - langue, territoire, religion-condamnés, croyait on, à s'atrophier et à disparaître.» (1991:1)

5. L'appropriation de l'espace résidentiel infléchit des types d'appartenance au quartier. Il est territoire d'appartenance pour l'habitant stable, il est territoire de référence normée pour le nouveau venu en promotion sociale terminale visant une acceptation dans le quartier, il reste lieu de référence nominale pour le « conquérant urbain » en quête de promotion sociale.

6. Cette transformation peut aussi être approchée par la théorie des statuts et des rôles sociaux.

7. Déjà de Tocqueville faisait remarquer que «Les institutions communales sont à la liberté ce que les écoles primaires sont à la science ; elle la mettent à la portée du peuple.» (1963:57) Mais il ajoute: «En rendant la commune forte et indépendante, ils [les gouvernants] craignent de partager la puissance sociale et d'exposer l'État à l'anarchie. Or, ôtez la force et l'indépendance de la commune, vous n'y trouverez jamais que des administrés et point de citoyens.» (de Tocqueville, $1963: 61$ ) 
8. Les modalités concrètes/abstraites caractérisent aussi des opérations également applicables au langage et au temps.

9. La valeur socialisante ou solidarisante de l'espace est une croyance démentie par de nombreux (Chamboredon et Lemaire (1970; Remy, 1975).

10. Une rue d'ambiance connaît (1) un temps fort d'expression dans la journée, (2) un lieu spécifique d'expression, (3) une gamme d'activité distinctes, (4) une densité de population assurant un fond d'anonymat, (5) une connotation affective variant selon les groupes de référence (Voyé, Remy, 1975 : 300).

11. Cette caractérisation de la ville explique pourquoi « la Ville ou la Cité sont les cadres préférés des réformateurs ou l'image la plus représentative du nouvel ordre social et politique de l'humanité transfigurée ». (Mucchielli, R, $1960: 176$ )

12. Voir l'analyse de la symbolique du changement de Marie J-L. (1989).

13. Voir, par exemple, les efforts entrepris dans l'aménagement des jardins pour les marquer d'histoire (De Roux, 1991 ; Lassus, 19).

14. A une emblématique municipale centrée avant tout au centre (...) a succédé une stratégie d'image visant l'espace communal tout entier, chargée d'en affirmer l'homogénéité interne et corrélativement d'en souligner autant que possible les différences de voisinage.» (Gaudin, 1989 : 72).

\section{RÉSUMÉS}

L'espace structure la communication en même temps qu'il est interprété et modifié par celle-ci. À tout au moins dans les formes de son appropriation par les êtres qui l'occupent. La dénomination même des différences composantes de la ville par ses habitants offre un exemple de la relation entre espace et communication en même temps qu'elle constitue un indicateur du sentiment d'appartenance.

\section{AUTEUR}

\section{PAUL STRYCKMAN}

Professeur à l'Université Laval où il enseigne les méthodologies de recherche. Sociologue, il fait notamment autorité en sociologie des religions. Il a mené des travaux importants aux États-Unis et au Mexique et est régulièrement professeur-invité aux Universités de Bordeaux et de Louvain la Neuve. 